\title{
Le point de vue de la psychanalyse : le plaisir oral est-il nutritionnellement correct ? ${ }^{1}$
}

\begin{abstract}
Michelle LE BARZIC
<michelle.le-barzic@wanadoo.fr>
\end{abstract}

\section{Le lait à la source du plaisir}

Au plaisir des mots...

Définir le plaisir n'est pas une mince affaire, surtout lorsque la question vous est posée par un enfant de moins de 3 ans face auquel le dictionnaire n'est d'aucun secours! En revanche, lorsque le recours en est autorisé par la maturité des interlocuteurs, on découvre que le plaisir réunit le corps et l'esprit désunis par la rationalité cartésienne, et que celui des psychanalystes ne s'écarte pas de celui des dictionnaires!

Dans l'édition 1874 du Littré, le plaisir est un "mouvement, sentiment plaisant, excité dans l'âme par une impression physique ou morale ». II siège donc dans l'âme et peut être provoqué aussi bien par le corps que par l'esprit. La succession des exemples de plaisir proposés par Littré est édifiante. Le premier est emprunté à Corneille et concerne celui de la vengeance... au féminin : "C'est un plaisir bien doux que de se voir vengée ». C'est un plaisir de l'âme. C'est ensuite La Fontaine qui nous met en garde contre la peur qui peut gâcher le plaisir : « Fi du plaisir que la crainte peut corrompre». Un avertissement que devraient méditer tous les agitateurs de risques sanitaires! Blaise Pascal, enfin, nous affirme que le plaisir est incontestablement la grande affaire de l'homme : « L'homme est né pour le plaisir ; il le sent, il n'en faut point d'autres preuves».

\footnotetext{
${ }^{1}$ Conférence prononcée dans le cadre du colloque "Le plaisir: ami ou ennemi de notre alimentation? ", organisé par l'IFN le 12 décembre 2006, et dont les actes complets seront publiés fin 2007.
}

\begin{abstract}
For human consumers, eating means more than getting nourishment. Alimentary pleasure is essential for nutritional and psychologic equilibrium in humans. The freudian oral stage is a stage of first importance for the personality built process. Cognitive learns, affects and social adaptation emerge in first years through nourishing learnings, during family exchanges. The human alimentary function transcends biology and participates in individual and collective humanization. Nutrition specialists must be aware of this complex reality.
\end{abstract}

Key words: oral pleasure, eating behaviour, learnings, nutritional and psychologic equilibrium, humanization

Au XXI ${ }^{\mathrm{e}}$ siècle, le mouvement a été remplacé par l'état et l'excitation par la satisfaction. La définition du Petit Larousse 2000 : «État de contentement que crée chez quelqu'un la satisfaction d'une tendance, d'un besoin, d'un désir, bien être », tient pour équivalents la psychologie du désir et la biologie du besoin dès lors qu'ils sont satisfaits. L'exemple proposé : « J'ai lu ce roman avec plaisir» inscrit l'activité intellectuelle parmi les sources du plaisir.

La version informatique du Petit Robert s'avère assez proche: "Etat affectif fondamental (affect), un des deux pôles de la vie affective, sensation ou émotion agréable, liée à la satisfaction d'une tendance, d'un besoin, à l'exercice harmonieux des activités vitales ». Elle ajoute les notions d'affectivité et d'exercice harmonieux des activités vitales. Avec Voltaire, la philosophie des Lumières fait du plaisir un devoir : " Le plaisir est l'objet, le devoir et le but de tous les êtres raisonnables ».

Au terme de ces définitions officielles, il apparaît que non seulement le plaisir n'est pas superflu, mais qu'il est essentiel à l'harmonie de l'existence humaine. C'est dire s'il est déraisonnable de « gâcher le plaisir »! Les experts de I'hygiène alimentaire doivent en être avertis !

\section{...Et de la chose}

Le couple plaisir/déplaisir est au cœur de la théorie psychanalytique et le plaisir oral est le socle du développement de la personnalité. Selon Freud, le plaisir émane de l'accomplissement de la pulsion alimentaire vitale. "C'est l'activité essentielle à la vie de l'enfant qui le lui a appris, la succion du sein maternel ou de ce qui le remplace. [...] l'excitation causée par l'afflux de lait chaud a provoqué le plaisir » [1]. C'est le lait chaud dans la bouche qui procure le plaisir originel. Le plaisir oral est donc le prototype, le modèle du plaisir à partir duquel tous les autres vont se déployer. Le plaisir est en outre une stratégie de la nature pour « arriver à ses fins »: sa recherche crée la motivation qui va pousser le mangeur à se procurer sa nourriture pour préserver son existence et, donc, celle de l'espèce. C'est l'excitation et l'apaisement de la tension désagréable qui vont provoquer le plaisir. Pour Freud, plaisir et sexualité sont synonymes (encadré 1).

\section{L'oralité humanisante}

Qu'elle soit organisée ou accidentelle, la faim est une violence qui peut susciter des réactions extrêmes, destructrices, évoquées par la littérature et la filmographie historiques. En procurant la paix des sens, la nourriture favorise I'harmonie des relations avec l'entourage. C'est pourquoi, pour l'homme, manger est plus que se nourrir, et l'alimentation humaine est partie prenante de l'humanisation des sociétés humaines depuis les origines.

En s'organisant socialement contre l'urgence nutritive, la fonction alimentaire humaine s'est progressivement complexifiée. Elle obéit à une triple finalité : nutritive, hédonique et symbolique. À l'instar des stades pulsionnels de la psychanalyse, ces trois finalités s'étayent les unes sur les autres et interfèrent intimement. Pour les besoins de la démonstration, nous allons les décrire isolément et successivement.

\section{Manger : un besoin vital}

Tout commence par la finalité nutritive. Pour pouvoir éprouver du plaisir, encore faut-il être 


\section{Encadré 1 \\ Quelques citations de Freud}

"Nous connaissons, en ce qui concerne la région bucco-labiale, les moyens dont se sert la nature pour arriver à ses fins : cette partie du corps sert en même temps à la préhension des aliments ».

"La succion nous a fait connaître les trois caractères essentiels de la sexualité infantile. [Celle-ci se développe en s'étayant sur une fonction physiologique essentielle à la vie] ».

"On peut donc dire que le but de la sexualité est de substituer à la sensation d'excitation projetée dans la zone érogène une excitation extérieure qui l'apaise et crée un sentiment de satisfaction. Cette excitation extérieure est le plus souvent une manipulation analogue à la succion ».

Sigmund Freud, La sexualité infantile, 1905

en vie. Manger est un besoin vital. Le petit mammifère humain nouveau-né se jette sur sa fontaine à lait sans état d'âme, tout comme le petit mammifère bovin, ovin ou porcin. Première étape de l'humanisation du mammifère omnivore humain, la période nourricière est une phase cruciale au cours de laquelle l'affectivité va progressivement se dégager de la primauté du physiologique sans pour autant l'annuler. Nous avons tous dans l'oreille le signal sonore par lequel un nourrisson manifeste sa faim, génératrice d'une tension interne qui lui fait éprouver la réalité tout entière $-y$ compris le monde extérieur environnant -, comme hostile et mauvaise. L'assouvissement de la faim restaure I'harmonie du monde. La satiété provoque l'apaisement intérieur promoteur du plaisir. Le monde redevient bon. Concomitante à la maturation du système nerveux, la répétition de ces séquences s'accompagne de l'identification de la personne habituellement dispensatrice de la satisfaction, la mère ou son substitut. Cette étape constitue les prémices de l'amour. On peut dire que l'amour pour la mère est un rejeton de la reconnaissance du ventre!

La personnalité et la socialisation vont s'élaborer à partir de cette séquence alimentaire originelle. Pour la psychanalyse, I'oralité ne se limite pas au buccal. Si la tétée est le paradigme de l'expérience « orale », elle s'accomplit dans un bain sensoriel composé de chaleur, de contacts, d'odeurs et de sons, qui pénètrent le corps de l'enfant en même temps que le lait. Le climat affectif dans lequel se déroulent les échanges est crucial pour l'épanouissement affectif ultérieur de l'enfant. La reconnaissance de la mère va diviser le monde en deux, celui des objets familiers - potentiellement bons - et celui des objets étrangers - dangereux sinon mauvais. Une fois la mère identifiée, sa seule présence - et non plus seulement le fait d'être nourri par elle - devient source de plaisir.

Au cours de son éducation nourricière, la mère va apprendre à l'enfant - par l'intermédiaire des " castrations symboligènes » [2] - que la nourriture n'est pas la seule source de plaisir possible. Elle va lui faire découvrir que la parole - les mots qui passent également par la bouche

Figure 1. Manger, un plaisir en soi.
Cette diapositive illustre en outre la multiplicité et la complexité des enjeux de l'apprentissage alimentaire. Poisson, haricot vert, pommes de terre, le contenu de l'assiette est conforme à la diversité nutritionnelle requise pour l'alimentation du mammifère omnivore humain. La situation "à table", l'usage de couverts et de l'assiette, qui distingue le comportement alimentaire humain de celui des autres animaux, illustre en outre le caractère socialisé, humanisé, de la fonction alimentaire humaine. L'apprentissage des manières de table, des règles du partage avec un nombre croissant de convives, est un terrain privilégié d'apprentissages sociaux. La convivialité est une clé pour apprendre le plaisir de partager avec les autres, mais peut-on partager un plaisir qui n'a pas été éprouvé individuellement?

\section{La finalité symbolique}

Le symbole, c'est ce qui relie plusieurs êtres autour d'un signifiant commun. Les sociétés se sont organisées autour de la symbolique alimentaire. L'apprentissage des manières de table de son groupe d'appartenance est une initiation aux règles du partage et aux manières de penser les relations des membres du groupe entre eux et avec le monde qui les entoure. C'est pourquoi, pour être «bonnes à manger ", les nourritures humaines doivent aussi être « bonnes à penser » [3].

Un enfant qui souffle les bougies de son gâteau d'anniversaire - le sien et pas un autre comprend que ce rituel marque non seulement le temps qui passe pour lui (les années qui s'ajoutent avec les bougies) mais, surtout, la place singulière qu'il occupe au sein de sa

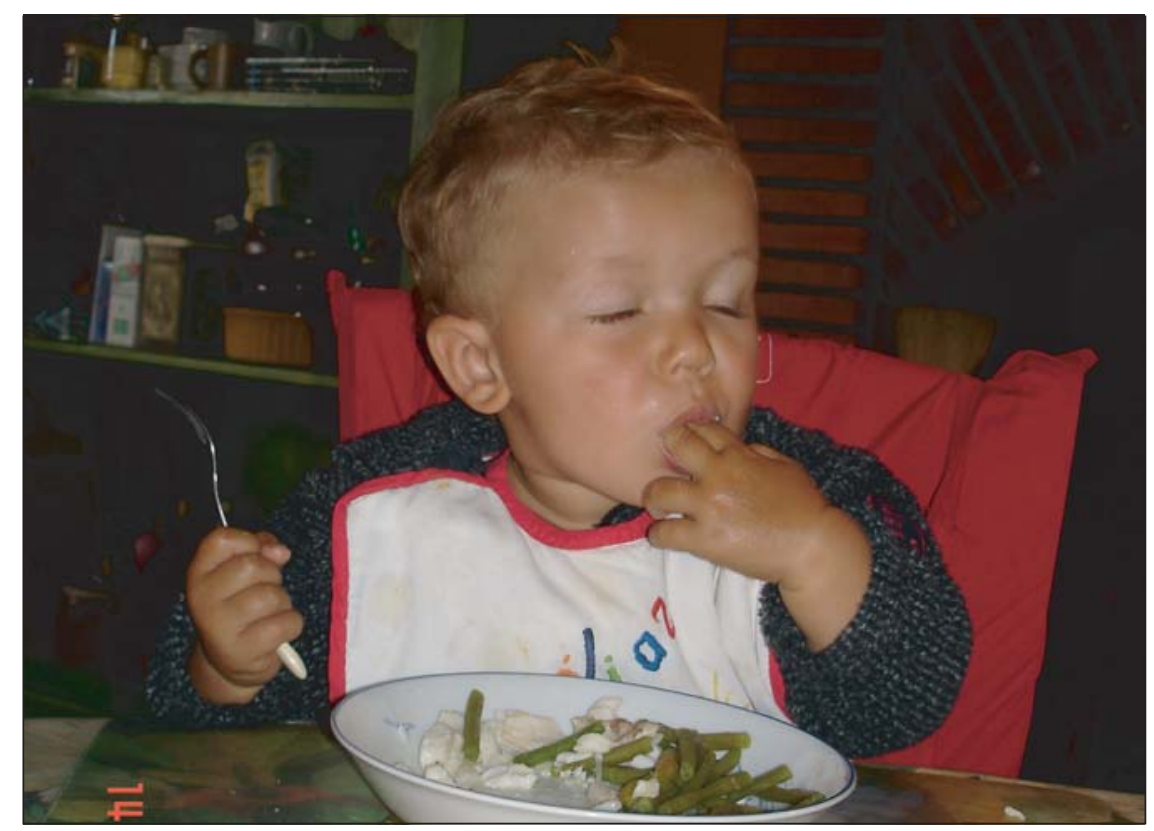


famille. En lui faisant mesurer son importance aux yeux des membres de sa famille, ce rituel est important pour développer l'estime de soi et la confiance en soi qui vont le soutenir dans l'avenir (figure 2).

Les repas en famille réunissent les sexes et les générations, séparés le reste du temps par des activités différentes. Les enfants y apprennent les rituels en vigueur dans leur groupe d'appartenance, les règles du partage et les préséances en vigueur dans leur niche de développement. L'apprentissage des règles familiales les initie aux règles de la vie en société. « Apprentissage, alimentation, sociabilité sont liés de façon étroite depuis longtemps, et constituent une sorte de noyau dur des cultures humaines" constate le psychosociologue Saadi Lahlou [4]. Sociabilité et apprentissage de I'alimentation sont indissociables et forment le creuset des civilisations humaines.

\section{Manger : une activité à risques}

Manger consiste à faire rentrer de l'étranger en soi. C'est, pour le moins, une activité «à risques »! Tout particulièrement pour l'omnivore humain qui n'a pas la chance d'un choix alimentaire forcé, prédéfini par la génétique. Claude Fischler a magistralement décrit I'angoisse fondamentale du mangeur qui découle du paradoxe de l'omnivore [5], tiraillé entre deux pulsions contraires: la néophilie pour assurer la variété indispensable à son équilibre nutritionnel et la néophobie pour se protéger du risque d'empoisonnement par des produits non comestibles (figure 3 ).

Pendant des millénaires, les traditions alimentaires religieuses, sociales et familiales ont protégé les mangeurs de cette angoisse d'incorporation en leur transmettant les connaissances de leurs ancêtres sur les aliments comestibles et les meilleures façons de les accommoder. Le développement des techniques agricoles était également une façon de se protéger de la famine, qui était le risque alimentaire majeur depuis les origines.

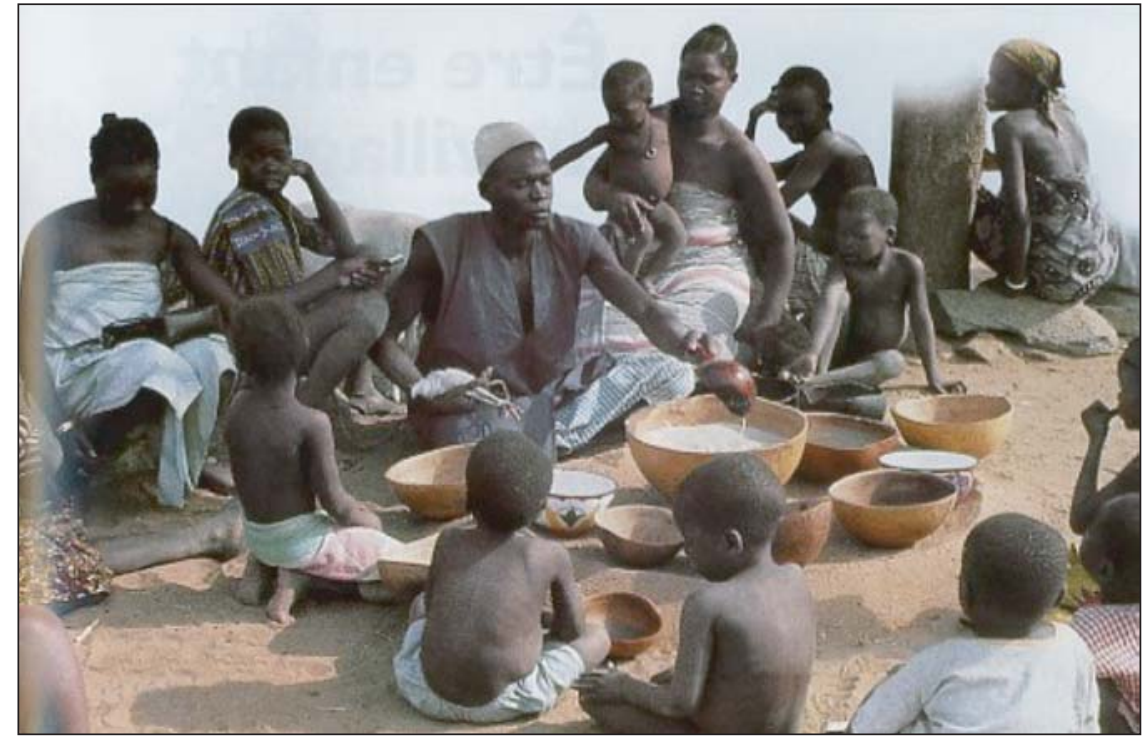

Figure 2. Un partage transgénérationnel.

L'angoisse originelle d'incorporation est aujourd'hui réactivée par les nouveaux risques alimentaires, conséquences de l'industrialisation qui réintroduit de l'inconnu dans la préparation des aliments dont le contrôle échappe aux mangeurs individuels. Les scandales alimentaires successifs ont encore accentué l'inquiétude consécutive à l'opacification par la technicité industrielle.

Plus encore, en protégeant les mangeurs occidentaux de la famine, I'industrialisation agroalimentaire a substitué les risques de la pléthore à ceux du manque. La vache grasse n'est pas seulement devenue "folle", elle induit des maladies qui s'apparentent à des châtiments pour avoir non seulement " assez à manger » mais plus que ce dont on aurait besoin. En Occident, aujourd'hui, le premier risque alimentaire est celui de grossir et l'obésité est devenue l'angoisse principale du mangeur. La crainte n'est plus tant que l'aliment incorporé soit mauvais, mais surtout qu'il « fasse grossir ». Les traditions religieuses ou régionales ont perdu leur pouvoir régulateur et rassurant.

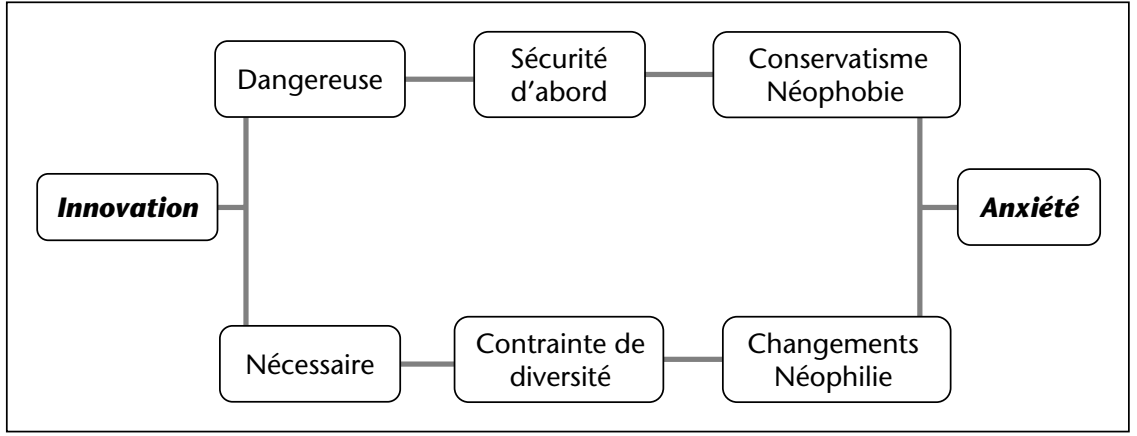

Figure 3. Le paradoxe de l'omnivore (d'après C. Fischler, L'Homnivore, 1990).
Quant aux habitudes familiales, elles ont totalement été disqualifiées, désignées comme responsables (et coupables!) des maux qui nous atteignent.

Nouveaux maux, nouveaux experts. Les mangeurs sont bombardés d'informations nutritionnelles aussi contradictoires et provisoires que péremptoires! La cacophonie diététique est entretenue par des cohortes d'experts autoproclamés qui persistent à ignorer l'ampleur de ce qu'ils ignorent. Inquiet pour lui et pour les siens, le mangeur ne sait plus à quel saint se vouer ni, surtout, comment s'y prendre pour appliquer ce qu'on lui dit aujourd'hui qui contredit ce que les mêmes lui affirmaient hier. Comment ne pas se décourager et ne pas s'abandonner aux tentations de l'instant? Comment la nourriture aussi diaboliquement représentée peut-elle rester "bonne à penser »?

\section{Le nutritionnellement correct est-il humainement convenable?}

Pour répondre au désarroi des mangeurs, la nutrition médicale a appliqué à l'obésité son raisonnement (supprimer le mal en éliminant sa cause) et ses outils (scientifiques et statistiques) qui avaient fait leurs preuves contre la maladie. Les seuils officiels d'IMC édités en 1997 constituaient une tentative d'encadrement des corps qui débordaient (tableau 1). Était-il légitime d'appliquer à l'alimentation et au corps humain le même raisonnement qu'à la maladie ? Relayant les critères esthétiques de minceur propres aux sociétés d'abondance, les risques sanitaires statistiquement associés au 


\begin{tabular}{|lcc|}
\hline Classification & IMC $\left.\mathbf{( k g} / \mathbf{m}^{\mathbf{2}}\right)$ & $\mathbf{k g} / \mathbf{1 , 7 0}$ \\
\hline Maigreur & $<18,5$ & $<53,4$ \\
Limites normales & $\mathbf{1 8 , 5 - 2 4 , 9}$ & $\mathbf{5 3 , 5}$ à $\mathbf{7 1 , 9}$ \\
Surpoids & $25,0-29,9$ & 72 à 86,4 \\
Obésité & $30,0-34,9$ & 86,4 à 100,9 \\
Obésité sévère & $35,0-39,9$ & 101 à 115,3 \\
Obésité massive & $>40,0$ & $<115,3$ \\
\hline
\end{tabular}

surpoids ont entraîné le glissement logique qui conduit à assimiler les seuils de corpulence à des seuils de santé. Aveuglée par le pouvoir qu'elle devait à la science, la médecine ne s'est-t-elle pas prise elle-même pour une science, perdant ainsi de vue son objet: la singularité clinique de la personne ? En prétendant dicter "des normes à la vie » [6], pour sacrifier à l'utopie de la santé parfaite [7], la Nutrition Médicale n'a-elle pas outrepassé les limites de sa compétence et abusé de son pouvoir ? En se focalisant sur les mensurations des mangeurs et les aspects nutritifs et énergétiques de la nourriture, ne méconnaît-elle pas I'aspect subjectif du corps, « où s'incarne la personne tout entière » [8], et la complexité de la fonction alimentaire humaine, «fait biosocial total » [4] ? En réduisant le corps humain à des normes statistiques de corpulence, la médecine encourage la norme de la minceur qui repose sur un déni massif de la réalité. Par définition, tous les humains ne peuvent pas rentrer dans les normes statistiques, que ce soit celles de I'OMS ou de l'esthétique en vogue. Trois milliards de femmes ne sont pas des top models (figure 4).

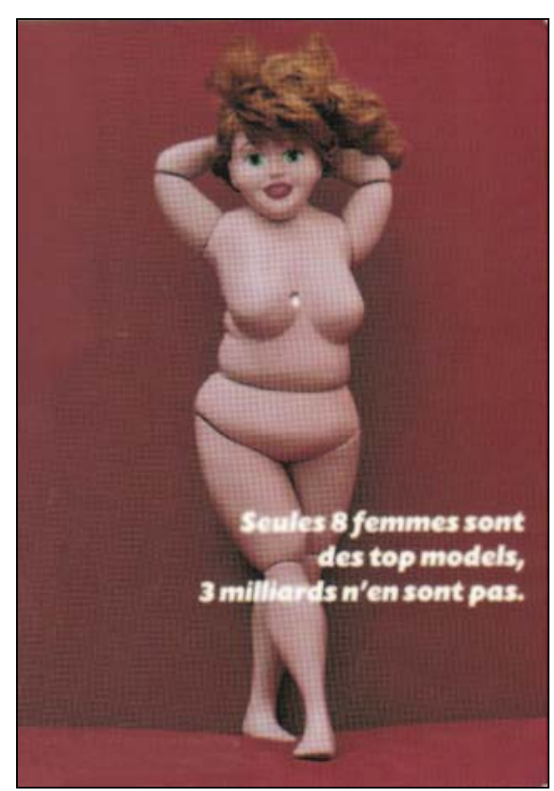

Figure 4. Trois milliards de femmes ne sont pas des top models.
Les normes médicales de corpulence reposent sur un autre déni : celui des conséquences de la modernité alimentaire. Celle-ci, depuis à peine plus d'un siècle, confronte le mangeur occidental à une réalité nouvelle : des sollicitations alimentaires démultipliées par l'industrialisation et la mondialisation agroalimentaires alors que les occasions de "brûler » les calories absorbées sont réduites par les transports motorisés, des logements et des vêtements plus chauds, en même temps qu'une sédentarisation croissante des comportements (télévision, ordinateur, etc.). Ayant plus à manger et moins à bouger, le mangeur occidental est logiquement appelé à peser en moyenne plus lourd que ses aïeux. Or, les normes de corpulence qui lui sont imposées sont inférieures à celles de ses prédécesseurs : des corpulences féminines, jugées superbes quand elles sont sculptées par Maillol ou peintes par Renoir, seront considérées comme obèses et esthétiquement décriées si elles sont croisées dans la rue!

Les mangeurs occidentaux du XXI ${ }^{\mathrm{e}}$ siècle sont confrontés à une véritable mutation de leur mes est inévitable. Elles concilieront les contraintes des invariants universels de la réalité humaine et celles du nouveau contexte. Encore faut-il leur en laisser le temps. La science et la philosophie s'associent pour dénoncer l'aberration que sont les normes théoriques imposées (encadré 2).

En érigeant la nourriture comme bouc émissaire de "l'épidémie d'obésité », le discours médical normatif dominant depuis plusieurs décennies a diabolisé la nourriture et, donc, le plaisir alimentaire. Ce déni n'est certainement pas étranger à l'aggravation de l'obésité et à la multiplication des troubles du comportement alimentaire, de la boulimie à l'anorexie mentale (dont la gravité est également déniée, aussi minimisée que les risques statistiques de l'obésité sont dramatisés), en passant par l'orthorexie et la restriction cognitive.

C'est la complexité de la fonction alimentaire qui est également déniée par la modélisation médicale, laquelle a tendance à ne s'en tenir qu'à la dimension nutritive et diététique de la nourriture en ignorant combien ses fonctions hédonique et symbolique sont fondamentaenvironnement. L'apparition de nouvelles nor- les : le plaisir gustatif et celui du partage s'enrichissent mutuellement. De ce déni découlent la diabolisation du plaisir alimentaire et la stigmatisation de celui qui est suspecté de $s^{\prime} y$ adonner ! Jean-Pierre Poulain dénonce la façon dont «I'idéologie médicale participe à la justification de la labellisation (donc de la stigmatisation) comme déviant et contribue à la dépréciation des obèses ». C'est ainsi que le mangeur est transformé en malade et le malade en coupable ! Or, la culpabilité tue le plaisir oral, comme cela est expérimentalement démontré [9] (figure 5).

Les courbes de plaisir et de culpabilité chez 37 jeunes femmes normopondérales après I'ingestion d'une pomme (en gris) comparées à celle de chocolat (en noir) figurent sur le graphique. Dès la cinquième minute, le chocolat provoque un plaisir nettement supérieur à celui de la pomme. Mais la courbe de culpabilité du chocolat est également beaucoup plus élevée et elle dure dans le temps. La pomme provoque peu de plaisir mais pas de culpabilité. Mais, après 90 minutes, le plaisir de la pomme est plus important que celui du chocolat, pour lequel la culpabilité domine le plaisir! Cette étude révèle que - comme dans la chanson - le plaisir du chocolat « ne dure qu'un moment » mais que son remords "dure toute la vie »! Les auteurs de l'étude concluent que la culpabilité au chocolat est probablement induite par les cognitions négatives reliées à la nourriture et qu'elle est déterminée par les attitudes culturellement induites vis-à-vis de la minceur et du poids.

De fait, il est exact que depuis plus de 50 ans, les mangeurs occidentaux sont bombardés $d^{\prime}$ « informations » sur les aliments qui sont «bons » et ceux qui sont « mauvais » pour sa santé, la santé étant, comme on l'a vu, réduite $\mathrm{au}$ « bon » poids qu'il faut peser. Les aliments considérés comme « bons à manger » (et donc «bons à penser ») sont donc avant tout « ceux qui ne font pas grossir », quelles que soient par ailleurs leurs vertus nutritionnelles. Ainsi qu'il a été démontré par Oakes, ces préjugés pris pour du savoir peuvent induire à l'insu des mangeurs les écarts diététiques qu'ils croyaient éviter [10] (figure 6).

Interrogés sur le pouvoir de faire grossir de 11 paires d'aliments composés, de valeur calorique équivalente, où l'un comporte un aliment réputé "sain » et l'autre un aliment réputé «mauvais», 182 sujets adultes minimisent l'effet pondéral du premier et majorent celui du second. Les messages de santé simplistes et manichéens en vigueur depuis des décennies sont responsables de ces croyances erronées qui risquent de conduire les mangeurs soucieux de leur poids et leur santé à abuser à leur insu, en toute bonne conscience diététique, 
des aliments ayant la « bonne » réputation de ne pas faire grossir !

\section{Le plaisir de manger se découvre en famille}

Le plaisir de manger ne se prescrit pas, pas plus qu'il ne s'enseigne. Il se découvre en famille, en partageant les repas familiaux. On ne naît pas " Humain », on le devient par l'éducation familiale, qui s'amorce dès les soins nourriciers. Le plaisir oral, éprouvé par le nourrisson puis dans les échanges familiaux, en particulier autour de la nourriture, est un facteur primordial de la pacification humanisante des relations humaines. L'identification aux comportements parentaux est un puissant facteur de développement des goûts alimentaires. Les repas familiaux sont les occasions privilégiées pour découvrir la nourriture, apprendre "à jouer » avec elle, se familiariser avec les aliments et surmonter les néophobies, ainsi que nous I'apprend Nathalie Rigal.

Le mode d'éducation alimentaire des parents, profondément influencé par leur propre comportement alimentaire, lui-même en relation avec leur image du corps et leur rapport au poids, retentit sur l'évolution pondérale de leur enfant, parfois de façon paradoxale [11] (figure 7).

Cette étude longitudinale menée tous les 2 ans, de 5 à 9 ans, chez 140 fillettes et leurs parents, montre qu'une éducation alimentaire restrictive de la part des parents peut induire chez des enfants qui n'ont pas de problème de poids au départ, un comportement alimentaire (manger au-delà de la faim) générateur de prise de poids. Les groupes sont constitués en fonction de l'IMC et de l'attitude maternelle : surpoids et forte restriction par la mère, surpoids et faible restriction par la mère, poids moyen et forte restriction maternelle et poids moyen sans restriction maternelle. Le comportement maternel perdure avec les années et, à 9 ans, on constate que la consommation des calories superflues (facteur d'augmentation de $\mathrm{I}^{\prime} \mathrm{IMC}$ ) des fillettes sans surpoids qui sont restreintes par leur mère se rapproche de celle des fillettes en surpoids et restreintes, alors que celle des fillettes en surpoids à 5 ans qui n'ont pas été restreintes est restée proche de celui des fillettes sans surpoids non restreintes. Cette étude démontre magistralement que le comportement parental, pourtant animé des meilleures intentions préventives, a plus d'influence sur l'évolution pondérale - par I'intermédiaire du comportement alimentaireque le poids initial.

La médicalisation du poids risque de perturber les interactions familiales. Être un «bon parent » cesse d'être le parent nourricier qui manifeste son amour en nourrissant son

\section{Encadré 2}

\section{Citations}

"Dans des situations différentes, il y a des normes différentes qui, en tant que différentes, se valent toutes. "

G. Canguilhem, 1966

"La plasticité métabolique montre qu'il faut se méfier des dogmes car ce qui est vrai un jour n'est pas forcément vrai le lendemain, que ce qui est vrai en Alaska n'est pas forcément vrai en Chine ».

X. Leverve, Colloque IFN Des Aliments et des Hommes, 2004

- 19 à 30 ans

- IMC 21,6 $\pm 1,8 \mathrm{~kg} / \mathrm{m}^{2}$

La culpabilité au chocolat est «probablement induite par les cognitions négatives reliées à la nourriture qui sont déterminées par les attitudes culturellement induites vis-à-vis de la minceur et du poids. »
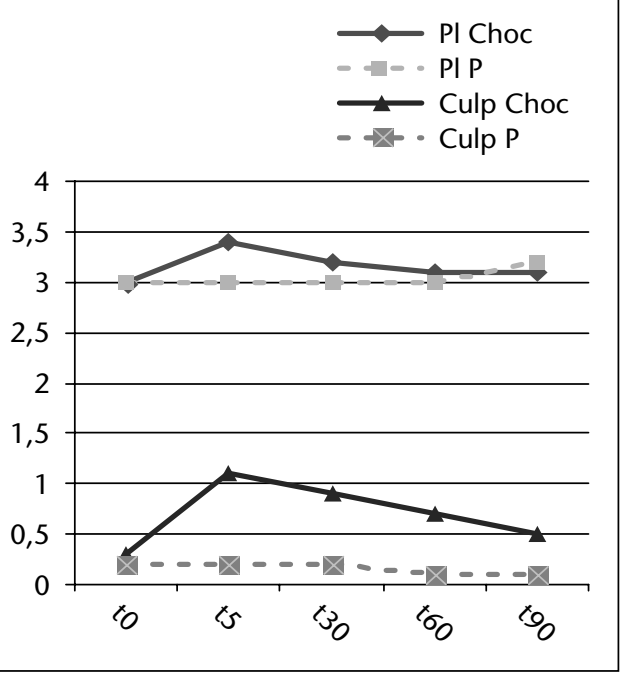

Figure 5. La culpabilité tue le plaisir oral (d'après Macht et Dettmer, Appetite, 2006).

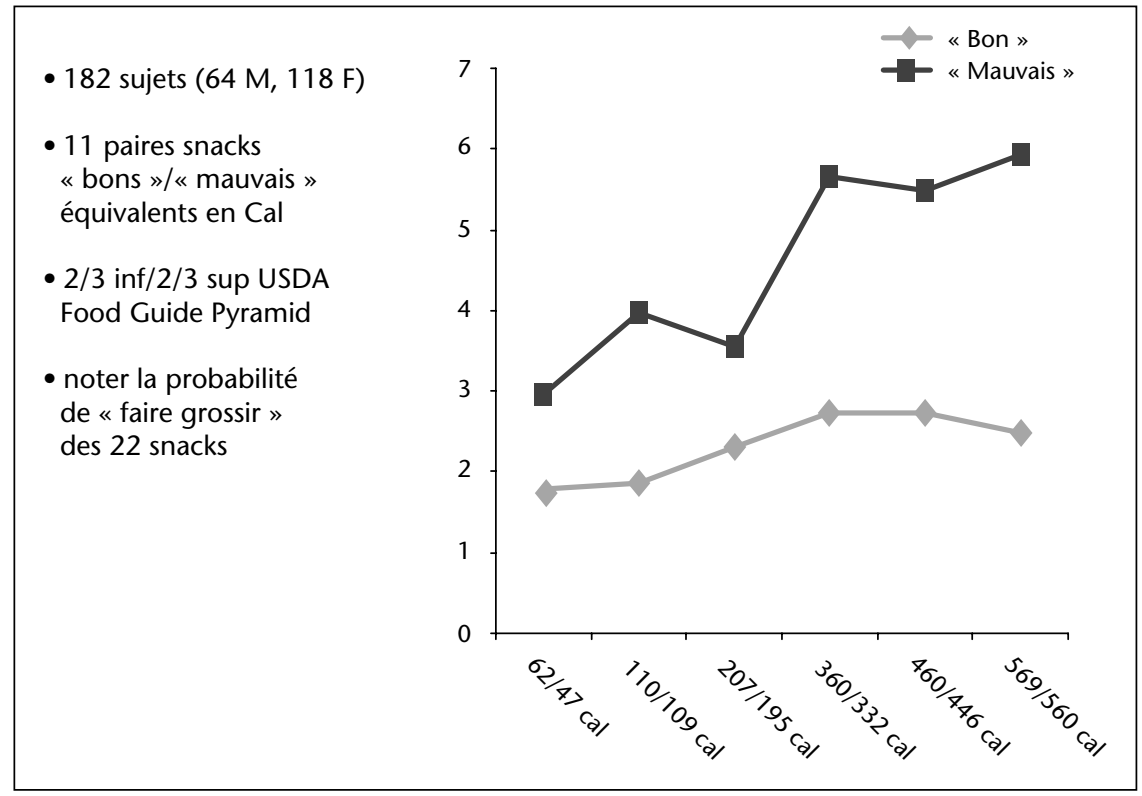

Figure 6. Les réputations trompeuses (d'après M. Oakes, 2005).

enfant ; cela devient " obéir à un autre », le médecin, qui devient le super «surmoi » alimentaire qui disqualifie les habiletés parentales héritées des aïeux. La convivialité familiale se trouve ainsi barrée par l'autorité médicale qui impose un modèle venu de l'extérieur au lieu du plaisir alimentaire inventé en famille, tous ensemble, au fil des jours.

Pour éviter ces écueils et restituer leur compétence alimentaire aux parents, un nutrition- 


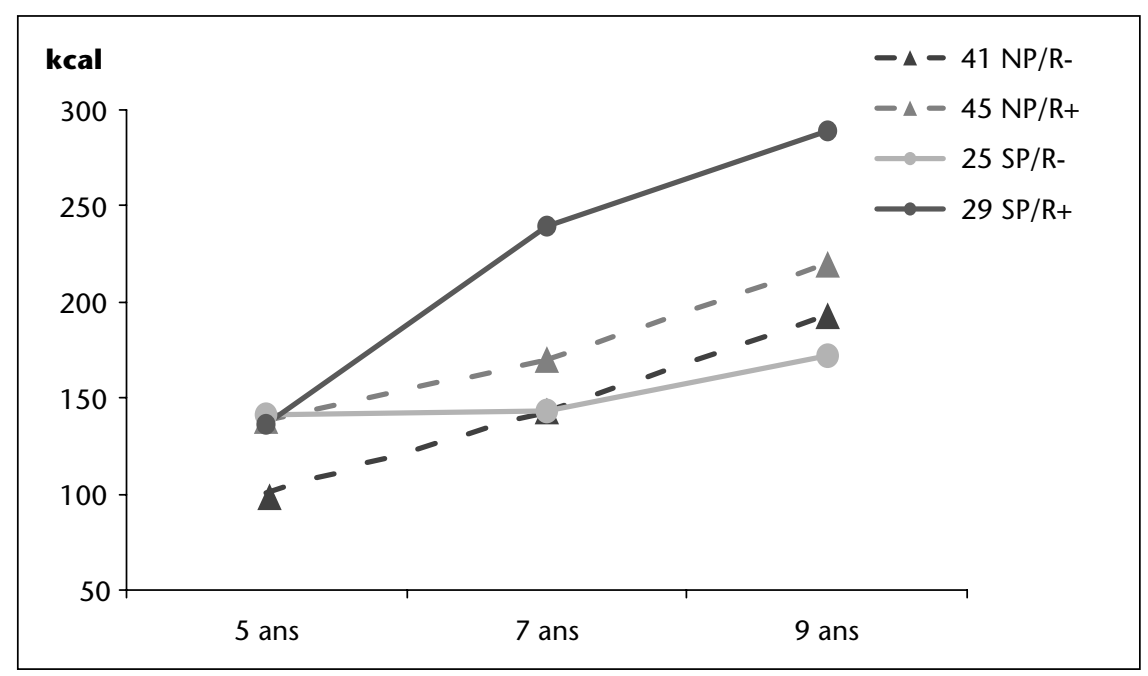

Figure 7. Restriction à 5 ans : calories superflues à 9 ans (d'après L.L. Birch et al., Ann / Clin Nut, 2003).

niste israélien [12] a développé des consultations réservées aux parents pour les aider à modifier leur comportement éducatif vis-à-vis de l'alimentation. Sept ans après, les résultats pondéraux chez les enfants sont très supérieurs à ceux obtenus chez les enfants qui avaient été impliqués dans des consultations traditionnelles (impliquant les enfants avec les parents).

\section{Conclusion}

"Si c'était si simple de manger moins et bouger plus pour peser moins, il n'y aurait plus un obèse en Occident ». Aussi évident qu'apparaisse cet aphorisme, le constat des pratiques quotidiennes suffit à nous convaincre qu'il n'est pas superflu de le répéter.

Reportons nous maintenant à la définition des conduites alimentaires par les anthropologues
Empruntons, en guise de conclusion, la formule de Claude Debussy à propos de la musique : "On peut vivre sans le plaisir oral, mais tellement moins bien!».

\section{RÉFÉRENCES}

1. FREUD S. La sexualité infantile. In : Trois essais sur la théorie de la sexualité (1905). Paris : Gallimard, 1962.

2. DOLTO F. L'image inconsciente du corps. Paris : Seuil, 1984.

3. LEVI-STRAUSS C. Le Totémisme aujourd'hui. Paris : PUF, 1962.

4. LALHOU S. Penser manger. Paris : PUF, 1998.

5. FISCHLER C. L'Homnivore. Paris: Odile Jacob, 1990.

6. CANGUILHEM G. Le normal et le pathologique. Paris : PUF.

7. SFEZ L. La santé parfaite. Critique d'une nouvelle utopie. Paris : Seuil, 1995.

8. MARZANO-PARISOLI MM. Penser le corps. Paris : PUF. domaine où la tradition et l'innovation importent autant I'une que l'autre, ou présent et passé se mêlent pour servir la nécessité de l'heure, fournir la joie d'un instant, convenir à la circonstance » [13]. Elles pacifient les relations en réunissant les contraires (tradition, innovation, présent et passé), tout en remplissant les trois finalités de la fonction alimentaire : biologique (la nécessité de I'heure), hédonique (la joie d'un instant) et symbolique et sociale (convenir à la circonstance).

J'espère vous avoir convaincu que La nourriture, c'est la vie, à laquelle, selon Georges Canguilhem on ne saurait dicter scientifiquement des normes [6]. Faut-il rappeller que la vie n'est pas une maladie et que le plaisir oral ne se prescrit pas, mais qu'il est important de ne pas lui faire obstacle comme tend à le faire l'idéologie du nutritionnellement correct [14]. ?
9. MACHT M, DETTMER D. Everyday mood and emotions after eating a chocolate bar or an apple. Appetite 2006 ; 46 : 332-6.

10. OAKES M. Stereotypal thinking about foods and perceived capacity to promote weight gain. Appetite 2005 ; 44 : 317-24.

11. BIRCH LL, FISCHER JO, DAVISON KK. Learning to overeat : maternal use of restrincting feeding practices promotes girls' eating in the absence of hunger. Am / Clin Nut 2003 ; 78 : 215-20.

12. GOLAN M, CROW S. Targeting parents exclusively in the treatment of childhood obesity : long term results. Obes Res 2004 : 357-61.

13. GIARD L. «Faire-la-cuisine ». In: de Certeau M, Giard L, Mayol P, eds. L'invention du quotidien. Paris : Gallimard, 1980.

14. LE BARZIC M, POUILLON M. La meilleure façon de manger. Paris : Odile Jacob, 1998. 